\title{
A Review on Neck Dissection
}

\author{
Dr.Shalini Singh ${ }^{1}$, Dr.Debasis Sahu ${ }^{2}$, Dr.A.V.Hemant ${ }^{3}$, Dr.U. Madhuri ${ }^{4}$, \\ Dr.Purva Sharma ${ }^{5}$, Dr.Vijay K.Thumpala ${ }^{6}$, Dr.Rahul VC Tiwari ${ }^{7}$. \\ ${ }^{I}(P G$ Student, Department of Conservative Dentistry, VYAS Dental College \& Hospital, \\ Jodhpur, Rajasthan, India) \\ ${ }_{2}^{2}$ (Senior Lecturer, Department of Oral \& Maxillofacial Surgery, Sree Sai Dental College \& \\ Research Institute, Srikakulam, Andhra Pradesh, India) \\ ${ }^{3,4}$ (Intern, Sree Sai Dental College \& Research Institute, Srikakulam, Andhra Pradesh., India) \\ 5 (Intern, VYAS Dental College \& Hospital, Jodhpur, Rajasthan, India) \\ ${ }^{6,7}{ }^{7}$ PG Student, Department of Oral \& Maxillofacial Surgery, SIBAR Institute of Dental \\ Sciences, Guntur, A.P, India)
}

\begin{abstract}
Surgery is the oldest and continues to be the most reliable form of treatment of malignancy. The treatment of the neck in patients with squamous cell carcinoma of the upper aero-digestive tract and other neoplasms of the head and neck region continues to be one of the most controversial issues in head and neck oncology. It is very crucial for an oral and maxillofacial surgeon to have a depth of knowledge in the surgical anatomy of neck, levels of lymph nodes, incisions, flaps, various types of neck dissections its indications, contraindications, advantages, disadvantages and complications. This review on neck dissection is published for providing the information in detail.
\end{abstract}

Keywords: Neck Dissection, History, Types, Techniques, Complications.

\section{Introduction}

Neck dissection is the removal of lymph nodes and lymph node bearing tissues of neck from the inferior border of mandible to the clavicle, as a treatment for head and neck malignancy. The rationale of treating squamous cell carcinoma which has metastasised to regional lymph nodes is based on the fact that spread by tumour emboli is the norm and that in transit metastasises. The resection of lymph node as part of the surgical management of head and neck cancer was initiated by crile (1906) and consolidated in a definite manner by hayes martin (martin et al 1951). The goal in the management of malignancy is tumour ablation either preservation of all vital and uninvolved structures. Neck dissection is either a component of a primary resection or a procedure unto itself. Ideally, radiation controls the primary disease, while the neck dissection is reserved for unresponsive cervical disease. Depending on the extent and location of the disease the appropriate procedure is chosen. Radical neck dissection has proved to be an extremely effective therapeutic measure and at present is probably the surgical procedure most frequently used in managing metastatic tumour suspected or actual in neck nodes. Here there is clearance of nodes from below the mandible to the clavicle involving submental, submandibular, jugular group of nodes and also nodes in the posterior cervical triangle. This makes the procedure a more multipurpose one. In recent years, however, a feeling has grown among many surgeons (bocca, 1975) that in certain circumstances radical neck dissection sacrifices structures unnecessarily and in an unselective manner.

The structures concerned are the spinal accessory nerve, internal jugular vein and the sternocleidomastoid muscle. Of all these, the spinal accessory is the most important functionally, its resection resulting in drooping of the shoulder from loss of upper trapezius function. The effect of loosing sternocleidomastoid muscle on one side is cosmetic rather than functional. Concern with the effect of loosing these structures, particularly the spinal accessory, has resulted in fresh evaluation of the whole surgical approach to lymph node resections, with the aim of preventing the sequelae without compromising the therapeutic effectiveness. These modified versions of radical neck dissection have been collectively referred to as 'functional' or 'modified' neck dissection. Neck dissection is indicated for metastatic disease in the superficial or deep cervical fasciae of the neck. Indications include cervical metastasis whose primary has recovered on treatment, cervical metastasis unresponsive to radiotherapy or chemotherapy and anticipated primary resection contiguous with occult or overt cervical metastasis. Neck dissection is contraindicated in disease beyond the superficial or deep cervical fascia. These include poor surgical candidate, rampant distant metastasis, base of skull disease, mediastinal or infraclavicular disease, unresectable or uncontrollable primary disease and extension into deep vital structures of neck. ${ }^{1}$ 


\section{Surgical Anatomy}

For doing neck dissection one should have thorough knowledge of the surgical anatomy of the neck. Neck dissection is actually a surgical dissection of the anterior and lateral neck done for the purpose of removing tumour and cervical lymph bearing tissues. A rational plan surgery for cancer of the cervical lymphatics is based on a thorough understanding and application of the detailed anatomy of the cervical lymphatic system, the deep cervical fasciae and the relationship of these to the muscles and neurovascular structures of the anterior and lateral neck. The neck can be divided into - anterior and posterior triangles about the sternocleidomastoid muscle. The anterior triangle is bordered by anterior border of the sternocleidomastoid muscle and by the inferior border of the mandible. The anterior neck is divided into two equal halves by the vertical midline from the mental symphysis to the suprasternal notch. The posterior triangle is bordered by the anterior border of the trapezius muscle, by the posterior margin of sternocleidomastoid muscle and by the middle third of the clavicle. The anterior triangle contains the cervical part of the aero digestive tract - larynx and trachea, hypo pharynx and oesophagus, thyroid and parathyroid gland; large neurovascular structure of carotid sheath; supra and infra hyoid muscles; associated lymphatic and neurovascular structures. It extends from the inferior border of the mandible cranially to the thoracic outlet of the chest caudally. The anterior triangle can be divided into submandibular triangle, sub mental triangle, carotid triangle and muscular triangle. The submandibular triangle is bordered superiorly by inferior border of the mandible, the two bellies of the digastric and the stylohyoid and mylohyoid muscles. The roof is by skin and platys. This contains the submandibular gland, associated fasciae, lymphatic structures (submandibular group of lymph nodes), part of anterior facial vein, facial artery and the marginal mandibular nerve branch of facial nerve. The deep part of the submandibular gland loops between mylohyoid and hyoglossus behind the posterior surface of the mylohyoid muscle. When the skin flap is elevated in this region, the skin flaps stops at the lower border of the mandible since the skin and the platys are leaving the operating field to overlie the facial muscles. The dissection is carried along the lower border of the mandible up to the attachment of the mylohyoid medially so that contents of the triangle can be dissected off the muscle. Submandibular gland is resected because of its associated lymph nodes. Facial vessels (artery \& vein) and the marginal mandibular nerve run in close approximation to the gland. The facial artery and vein come to lie alongside one another at the lower border of mandible, nearer the angle and it is here the vessels are sectioned. The veins stays superficial to the gland, running downwards and backwards deep to platysma and the mandibular division of facial nerve. It unites with the anterior branch of the retromandibular vein to form the common facial vein which joins with internal jugular vein. The artery passes upwards towards the mandible from the external carotid approaching the triangle deep to the gland, loops over it to come alongside the vein at the lower border of the mandible. Omohyoid muscle demarcates the carotid and the muscular triangle. In the carotid triangle the carotid vessel lie superficial. The lower and anterior part of the neck is the muscular triangle which contains the infrahyoid strap muscles of neck, the aerodigestive tract and the thyroid complex. It is bounded by the borders of the sternocleidomastoid muscle, trapezius muscle and the middle third of the clavicle. The roof is by the platysma and the skin.

The platysma cover is present only in the anterior part of the triangle, so dissection of the skin flap in the posterior part of the triangle is bit difficult. Floor is formed by prevertebral muscles - the scalene, levator scapulae and splenius which are covered by prevertebral fascia. The contents of this triangle include fibrofatty lymphatic containing tissue, cranial nerve xi, superficial and cutaneous components of the cervical nerve plexus and a host of small vascular bundles. The presence of which at surgery has in the past led to the description of this surgical area as "bloody gulch". The omohyoid present just above the clavicle in the lower part of the triangle marks out a small subclavian triangle. It has important surgical implication as in its depth are found the cervical and thoracic outflow of nerves and vessels into the axilla ( the brachial plexus from the inter-scalene interval ) and the subclavian vessels arching over the first rib from the thorax to the axilla. The transverse cervical artery a branch of the thyrocervical trunk runs from medial to lateral over the floor of the triangle, reaching the anterior border of the trapezius at the same point as the spinal accessory nerve. Transverse cervical vein is less constant in its relation. Artery lies between the fat that fills the triangle and the prevertebral fascia covering the floor of the mouth. The vein runs through the fat itself. In relation to omohyoid usually transverse cervical vein lies deep but sometimes it is superficial and joins the external jugular vein. Parallel to the transverse cervical vessels at the lower ends are the subclavian vessels. The anatomical structures encountered during routine neck dissection are the sternocleidomastoid muscle, spinal accessory nerve, trapezius, internal jugular vein, carotids, hypoglossal nerve, cervical fasciae and the lymphatic of cervical region. The sternocleidomastoid muscle is attached cranially to the lateral surface of the mastoid process of the temporal bone and to the lateral part of the superior nuchal line of the occipital bone. Caudally it divides into the sternal and clavicular heads to attach on the sternum and the clavicle. It is invested by superficial layer of the deep cervical fascia, which splits into two laminae to cover the superficial and deep surfaces of this muscle. At the anterior margin of the sternocleidomastoid muscle, thickened fascia attaches to the angle of the mandible forming an angular band "charpy's band". This band is tough and it is difficult to open into the interval between 
the sternocleidomastoid muscle, angle of the mandible and the parotid gland. This band should be sectioned. Crossing the outer surface of the sternocleidomastoid muscle in its upper half is the external jugular vein, marking out its course from the angle of the mandible to its midpoint of the posterior margin of the sternocleidomastoid muscle. Within one finger-breadth cranial and parallel to the vein, the great auricular nerve crosses the posterior surface of sternocleidomastoid muscle from erb's point (the midpoint along the posterior margin of the sterrnocleidomastoid muscle). From this point more nerves derived from the cervical plexus emerge and distribute the cutaneous part of anterior neck. The spinal accessory nerve can be identified entering the deep surface of the sternocleidomastoid muscle $4 \mathrm{~cm}$ or more below the mastoid process. It can be located at the erb's point just superior to where the greater auricular nerve surfaces from the deep neck. It can also be identified deep to trapezius which is about $2 \mathrm{~cm}$ finger width above clavicle. Its identification is important when preservation is planned as in modified neck dissection. Functionally sternocleidomastoid muscle aids in elevation of the thoracic cage and shoulder girdle, fixation of the limb, aid in lateral flexion of the head to the shoulder and rotate the head to direct the chin upward to the opposite side. Trapezius is one of the several muscles that elevate the shoulder girdle and retract the girdle medially. So preservation of the spinal accessory is vital for this function. Internal jugular vein trunk is part of the complex of veins, arteries, nerves, lymphatics and lymph nodes which run the length of the neck in a trough between the prevertebral muscles behind and the laryngopharynx medially. From the skull base to the root of the neck the internal jugular vein receives venous tributaries from the parotid, floor of the mouth, occipital region and from thyroid. It has no posterior tributaries. Each tributary has to be contained by ligature for removal of internal jugular vein in radical or modified neck dissection. Nodal tissues are found on the anterior, lateral and posterior aspects of the connective tissue sheath along the internal jugular vein. These are present posterolateraly to carotid sheath and these are removed along with internal jugular vein in radical neck dissection. Although covered by lymph nodes internal jugular vein lies within its own fascia and advential covering. This helps in preserving the vein as in modified or conservative neck dissection. Internal jugular vein drain the brain and the deep structures of the head and neck. So bilateral ablation of internal jugular vein results in venous stasis and massive cyanotic swelling of the face. Morfit and perzik 1952 demonstrated a case with simultaneous internal jugular vein ablation. They noted that swelling and venous engorgement subsided following the development of alternative venous channels. The common carotid branches of in the anterior triangle of the neck into the internal and external carotid arteries. Internal carotid proceeds straight and unbranched to skull base into the carotid canal through the carotid sheath. The external carotid gives branches in neck.

The superior thyroid artery arises below the greater cornu, the lingual artery arises below the level of digastric and the ascending pharyngeal arises posteriorly. The facial and maxillary arteries arise anteriorly deep to digastric muscle while occipital artery arise posteriorly. These arteries might sometimes need to be secured in the course of neck dissection. The ascending pharyngeal artery which arises at the junction of common carotid artery runs straight posteriorly to occipital region is usually not encountered in neck dissection. The hypoglossal nerve (cranial nerve xii) emerges between the internal jugular vein and carotid artery to descend in the lateral groove between them, to the point above carotid bifurcation. Here it gives off the superior root of the ansa cervicalis (the descendans hypoglossali of the ansa hypoglossi) and curves forwards across the lateral surface of both internal and external carotid artery. Then the nerve runs forward deep to stylohyoid and posterior belly of digastric and enters the posterior part of the submandibular triangle, where it lies on the lateral aspect of the hyoglossus muscle. It passes forward on the hyoglossus and disappears behind the posterior border of the mylohyoid muscle. It enters geinoglossus and supplies muscles of tongue except palatoglossus (via vagus). The brachial plexus is formed by the union of the ventral rami of the lower four cervical nerves and the greater part of the ventral ramus of the first thoracic nerve (c5-8 and t1). In the neck it lies in the inferior aspect of the posterior triangle in the angle between the clavicle and the lower part of the posterior border of the $\mathrm{scm}$. It enters the posterior triangle between the anterior and middle scalene muscles along with the subclavian artery which lies anterior and inferior to it. The plexus is crossed by the supraclavicular nerves, the nerve to the subclavius, inferior belly of the omohyoid, the external jugular vein and the transverse cervical artery. The phrenic nerve provides the sole innervation to diaphragm. It is comprised of fibers from cervical roots 3-5. It runs obliquely toward the midline along the anterior surface of the anterior scalene muscle and is covered by prevertebral fascia. The vagus nerve and the sympathetic trunk should not be confused for the phrenic nerve. The vagus lies anterior to the phrenic in the posterior aspect of the carotid sheath and the sympathetic trunk lies medial and posterior to the carotid sheath. Neither crosses over the anterior scalene. When transecting cervical rootlets as is routine in neck dissections, it is important to cut distal to their contributions to the phrenic. This can be accomplished by identifying the cervical nerves as they exit the plexus, and then transect them high on the specimen. The thoracic duct conveys the greater part of the lymph back into the circulating blood. It drains lymph from the entire body except for the right side of the head and neck, the right upper extremity, right lung, right heart, and part of the convex surface of the liver. The duct proper begins at the upper end of the cisterna chyli near the lower thorax through the aortic opening of the diaphragm. It then ascends through the posterior mediastinum with the aorta on its left and azygos vein on its right. The duct begins to course toward the left and 
enters the superior mediastinum, and ascends to the thoracic inlet along the left side of the esophagus. As the duct passes into the neck it lies anterior to the vertebral artery and vein, the sympathetic trunk and the thyrocervical trunk. It is separated from the phrenic nerve by the prevertebral fascia. Finally it descends in front of the first part of the left subclavian artery and ends by opening into the junction of the left subclavian and internal jugular vein. It may enter the distal ijv as well. It may be a single large duct of break up into a variable number of smaller vessels just prior to its termination. It is important to remember that there is a smaller lymphatic duct in the right neck. It descends along the medial border of the anterior scalene and ends by opening into the junction of the right subclavian and internal jugular veins. The preferred method to prevent chyle leak is the en bloc ligation of the lymphatic pedicle in which the lymphatic ducts lie. This should be done only after the carotid artery, vagus, phrenic, and ijv or identified. A leak may be difficult to detect, as chyle is not milky colored in the fasting individual. Microscopic examination may be of benefit if a leak is suspected. Having the anesthesiologist valsalva the patient at this time may increase the flow of chyle from a leaking duct facilitating its discovery. Digastric, stylohyoid and mylohyoid determine the neck dissection in the upper neck (submandibular area). The digastric muscle is attached to the deep surface of the mastoid in the digastric groove as a posterior muscle belly, to lesser cornu of the hyoid bone as a tendon slip and to the deep surface of the mandible in the mental region as an anterior belly. Associated with tendinous attachment of digastric to hyoid is the stylohyoid which splits it into two muscle bands. All major structures crossing between the head and neck pass deep to the posterior belly of digastric-stylohyoid complex.

These include the hypoglossal nerve, vagus nerve, carotid artery and internal jugular vein. Only the facial vessels and marginal mandibular nerve lie superficial in the submandibular region. This nerve has to be protected during neck dissection. The suprahyoid muscles lying between the hyoid bone and the body of the mandible constitute a muscular framework of the mouth (oral diaphragm). These include anterior digastric, geniohyoid and mylohyoid. They depress the mandible. Mylohoid is a sheet of muscle contributing to the floor of the mouth which is attached to the mylohyoid line on the inner aspect of mandible, the hyoid bone and to the muscle of opposite side by way of a midline muscular raphe. The deepest part of submandibular gland and the duct enters the floor of mouth by passing around the posterior or free margin of mylohyoid muscle. In the loose connective tissue above the mylohyoid there is the deep part of the submandibular gland, sublingual gland and ducts and hypoglossal nerve. The nerve to the anterior belly of digastric and mylohyoid muscles the mylohyoid branch of mandibular nerve is quite superficially placed in the suprahyoid region between the two muscles and can be injured during neck dissection. Its injury results in loss of muscular support to the floor of mouth and reduced tongue thrust in the first phase of swallowing. The fascia and fascial planes of the head and neck were described in detail in the 1930s by coller \& yglesius (1937) and by grodinsky \& holyoke (1938). Fascia and fascial planes are important in neck dissection as these help in recognising proper tissue planes. The fascia of the head and neck include superficial fasciae and deep fasciae. The deep fasciae is condensed in many layers as investing layer, pretracheal layer, prevertebral layer and carotid sheath. The superficial fascia of the anterior neck contains the platysma muscle. A plane can be developed superficial to platysma muscle or a skin flap can be raised to include the platysma muscle in it. Investing layer of deep fascia. Deep to superficial fascia lies a discrete fascial lamina, the investing layer of the deep cervical fascia which invests all around the neck in a collar like fashion. This fascia attaches dorsally to the cervical spine, cranially to the occiput, mastoid and inferior border of mandible. It invests around the parotid and submandibular gland. It is also attached to the hyoid bone, clavicle and sternum caudally. It invests around the sternocleidomastoid and trapezius muscle. In the anterior suprasternal region it attaches in the anterior and posterior side of sternum creating an inter-fascial space "space of burn" through which anastomotic superficial vessels cross the midline of the neck. Carotid sheath and pretracheal fascia. The investing layer of the deep cervical fascia has deeper extensions of connective tissue which invest around the carotid artery, internal jugular vein and vagus nerve known as carotid sheath. Multiple laminae of deep cervical fascia invest the suprahyoid and infrahyoid muscles and can be identified as thin muscle fascial laminae.

The deepest of these are associated with thyrohyoid muscle, thyroid gland and trachea. They invest the cervical part of airway and referred to as pretracheal fascia or middle layer of deep cervical fascia. Lateral extensions of this fascia gives rise to carotid sheath. The carotid sheath is continuos with the laminae of the neighbouring fasciae: that of pharyngeal wall, prevertebral muscles and fascia, sternocleidomastoid muscle and investing layer of deep fascia. Areolar connective tissue external to carotid sheath contains lymphnodes on the anterior, lateral and posterior aspects. Prevertebral fascia is the deepest cervical fascia invests prevertebral muscles. It extends from base of skull to the tubercles of the transverse process of all cervical vertebrae and is continuos along the vertebral surface of the vertebral column into the posterior mediastinum. It is recognised as the investment for splenius capitis; levator scapulae; anterior, middle and posterior scalene muscles; longus colli and capitis muscles and for nerve structures. The cervical and brachial plexus are invested by the prevertebral fascia which form the floor of the posterior cervical triangle of the neck. Neck dissection is done superficial to this plane to avoid damage to the cervical, brachial plexus and phrenic nerve. Lymphnodes and lymphatics of head \& neck are not visualised as such in a neck dissection. The procedure of neck dissection is designed to 
remove them as part of the overall specimen encased in the surrounding tissues, be it fascial covering, muscle or salivary gland. Occasionally larger lymphatics are recognised as distinct channels in the lower part of the neck but more often they are noticed when they are divided and clear fluid is seen to well up into the wound. The thoracic duct is at risk when dissection is carried low down on the left side of the neck. Greenfield and gottleib (1956) showed variations in the termination of the thoracic duct as in internal jugular vein, subclavian vein, external jugular vein, innominate vein and right internal jugular vein. The cervical lymphatic system is divided into superficial and deep chains. Superficial lymphatics perforate the first layer of cervical fascia to empty into deep cervical lymphatic chain. Their involvement requires ablation of large area of skin. The deep cervical lymphatics are important as they receive lymph from mucous membranes lining the mouth, pharynx, major salivary glands, thyroid and as well as the skin of head and neck. The deep cervical lymphatics accompany the internal jugular vein and their branches or lie within the major salivary glands. The deep cervical lymphatics along the internal jugular vein are grouped into jugulodigastric (subdigastric), juguloomohyoid (middle jugular) and lower / inferior jugular nodes. Lindberg \& jene 1972 showed that the most common areas involvement of the cervical lymphatic chain are the jugulodigastric or the junctional nodes of fisch. Internal jugular vein is sectioned between the upper and lower limits of the jugular group of lymph nodes and are removed along with the nodes. Lymph nodes of the posterior triangle are designated as upper, middle and inferior cervical nodes or spinal accessory group of cervical lymphatics. From metastatic point, this group of lymphnodes receive drainage from the nasopharynx, in the upper portion it receives drainage from the subdigastric group of deep cervical lymphnodes. In the submandibular area there are three group of nodes preglandular, interglandular and pre \& retro vascular nodes.

They drain the lower lip, cheeks, alveolar region, floor of the mouth and anterior tongue. They empty into the deep jugular chain of lymphatics. Preglandular nodes are present at the anterior to submandibular gland, interglandular lymphnodes are present within the gland while pre and retro vascular lymphnodes are present just anterior and posterior to facial vessels at the lower edge of the mandible. These group of lymphnodes are involved in metastatic cancer of oral cavity. The lower parotid nodes are present in the lower pole of the parotid gland and may be involved secondarily to the preauricular parotid nodes in cancer of parotid gland or in cases of the cancer of the upper lip. So in neck dissection this tail of parotid gland is removed along with the inferior parotid lymphnodes in this area. Superior jugular or jugulodigastric drain the posterior facial region including the tonsil. Middle jugular nodes drain middle part of aerodigestive tract and thyroid. Inferior jugular nodes drain the thyroid and cervical part of aerodigestive tract (oesophagus and trachea). ${ }^{1}$

\section{Lymph Node Levels}

\section{From Surgical Point Of View Lymphnodes Of Head And Neck Can Be Divided Into Nodal Regions:}

Level i: lymph node groups - submental and submandibular. Level ia is submental triangle. Its boundaries are anterior bellies of the digastric muscle and the hyoid bone. Level ib: submandibular triangle. The boundaries are body of the mandible, anterior and posterior belly of the digastric muscle. ${ }^{2}$

Level ii: lymph node groups - upper jugular. The boundaries are lateral border of the sternohyoid muscle anteriorly, posterior border of the sternocleidomastoid muscle posteriorly, skull base superiorly and level of the hyoid bone (clinical landmark) or carotid bifurcation (surgical landmark) inferiorly

Level iia and iib are arbitrarily designated anatomically by splitting level ii with the spinal accessory nerve.

Level iii: lymph node groups - middle jugular. Its boundaries are lateral border of the sternohyoid muscle anteriorly, posterior border of the sternocleidomastoid muscle posteriorly, hyoid bone (clinical landmark) or carotid bifurcation (surgical landmark) superiorly, cricothyroid notch (clinical landmark) or omohyoid muscle (surgical landmark) inferiorly.

Level iv: lymph node groups - lower jugular. Its boundaries are lateral border of the sternohyoid muscle anteriorly, posterior border of the sternocleidomastoid muscle posteriorly, cricothyroid notch (clinical landmark) or omohyoid muscle (surgical landmark) superiorly, clavicle inferiorly. Level iva denotes the lymph nodes that lie along the internal jugular vein but immediately deep to the sternal head of the scm. Level ivb denotes the lymph nodes that lie deep to the clavicular head of the scm.

Level v: lymph node groups - posterior triangle. Its boundaries are posterior border of the sternocleidomastoid muscle anteriorly, anterior border of the trapezius muscle posteriorly and clavicle inferiorly. Level va denotes those lymphatic structures in the upper part of level $\mathrm{v}$ that follow the spinal accessory nerve. Level vb refers to those nodes that lie along the transverse cervical artery. Anatomically, the division between these to subzones is the inferior belly of the omohyoid muscle.

Level vi: lymph node groups - [prelaryngeal (delphian), pretracheal, paratracheal, and precricoid (delphian) lymph nodes] - also known as the anterior compartment. Its boundaries are carotid sheath laterally, hyoid bone superiorly, and suprasternal notch inferiorly.

Level vii: lymph node groups - upper mediastinal. Its boundaries are carotid arteries laterally, suprasternal notch superiorly and aortic arch inferiorly 
Supraclavicular zone or fossa: relevant to nasopharyngeal carcinoma. Its boundaries superior margin of the sternal end of the clavicle, superior margin of the lateral end of the clavicle, the point where the neck meets the shoulder.

\section{Staging Of Neck Nodes}

The " $n$ " or nodal classification for cervical metastasis is consistent for all mucosal sites except the nasopharynx. Thyroid and nasopharyngeal carcinomas have unique nodal classifications that are based upon tumor behavior and prognosis. The staging systems for cervical metastases have been established by the american joint committee on cancer most recently updated in 1997 . $^{3,4}$

Regional lymph nodes (n)

Lip, oral cavity, oropharynx, hypopharynx, larynx, trachea, paranasal sinuses, major salivary glands

$\mathrm{Nx}$ regional lymph nodes cannot be assessed

N0 no regional lymph node metastasis

N1 single ipsilateral lymph node $3-6 \mathrm{~cm}$

N2 N2a single ipsilateral lymph node $3-6 \mathrm{~cm}$

$\mathrm{N} 2 \mathrm{~b}$ multiple ipsilateral nodes $<6 \mathrm{~cm}$

$\mathrm{N} 2 \mathrm{c}$ bilateral lymph nodes $<6 \mathrm{~cm}$

$\mathrm{N} 3$ any node $>6 \mathrm{~cm}$

Nasopharynx

Nx nodes cannot be assessed

N0 no regional lymph node metastasis

$\mathrm{N} 1$ unilateral metastasis in lymph nodes $<6 \mathrm{~cm}$ above the supraclavicular fossa

N2 bilateral metastasis in lymph nodes $<6 \mathrm{~cm}$ above the supraclavicular fossa

N3 metastasis in a lymph node(s)

$\mathrm{N} 3 \mathrm{a}>6 \mathrm{~cm}$

$\mathrm{N} 3 \mathrm{~b}$ extension to the supraclavicular fossa

Thyroid

$\mathrm{Nx}$ regional lymph noses cannot be assessed

N0 no regional lymph node metastasis

N1 regional lymph node metastasis

N1a metastasis in ipsilateral cervical lymph node(s)

$\mathrm{N} 1 \mathrm{~b}$ metastasis in bilateral, midline, or contralateral cervical or mediastinal lymph node(s)

\section{Classification Of Neck Dissection}

\section{American Academy Of Otolaryngology/Head And Neck Surgery Classifiaction}

In 1991, the committee for head and neck surgery and oncology of the american academy of otolaryngology/head and neck surgery developed a system for the classification of neck dissections. The academy's system remains the most widely accepted and has been endorsed by the american society for head and neck surgery. It is based on the following concepts:

1. Radical neck dissection is the standard basic procedure for cervical lymphadenectomy against which all other modifications are compared.

2. modifications of the radical neck dissection which include the preservation of any non-lymphatic structures are referred to as modified radical neck dissection (mrnd)

3. any neck dissection that preserves one or more groups or levels of lymph nodes is referred to as a selective neck dissection.

4.4)an extended neck dissection refers to the removal of additional lymph node groups or non-lymphatic structures relative to the radical neck dissection.

The following are the four major types and subtypes of neck dissections proposed by the academy: 1) radical neck dissection (rnd), 2) modified radical neck dissection (mrnd), 3) selective neck dissection (snd): a. Supra-omohyoid type, b. Lateral type, c. Posterolateral type, d. Anterior compartment type, 4) extended radical neck dissection. In 1989 medina suggested that the term "comprehensive neck dissection" be used whenever all the lymph nodes contained in levels $\mathrm{i}$ through $\mathrm{v}$ have been removed. The radical neck dissection and the modified radical neck dissection would, therefore, be included under this title. He then recommended three subtypes of modified radical neck dissection. ${ }^{4,5}$ 


\section{Medina's Classification}

1) comprehensive neck dissection: radical neck dissection, modified radical neck dissection- type $\mathrm{i}$ (xi preserved), type ii (xi, ijv preserved) and type iii (xi, ijv, and scm preserved) 2) selective neck dissection (a-d as above). In 1994, spiro recommended that the term "radical neck dissection" be used for any neck dissection in which four or more lymph node levels are removed, and that the term "modified radical neck dissection" be included under the heading of rnd. Additionally they included two other types of neck dissection: selective and limited. ${ }^{4}$

\section{Spiro's "Three-Tiered" Classification}

Radical (4 or 5 node levels resected), Conventional radical neck dissection, Modified radical neck dissection, Extended radical neck dissection, Modified and extended radical neck dissection, Selective (3 node levels resected), Supraomohyoid neck dissection, Jugular dissection (levels ii-iv), Any other 3 node levels resected, Limited (no more than 2 node levels resected), Paratracheal node dissection, Mediastinal node dissection, Any other 1 or 2 node levels resected The academies system for classification of neck dissection has become the most widely accepted and utilized system throughout the world. ${ }^{5}$

\section{Incisions}

There are various incisions used for neck dissections. Selection of incision for neck dissection is important to avoid complications such as wound break down, skin flap necrosis, exposure of the carotid artery following neck dissection. So it is essential to place incision properly being mindful of the vascular anatomy of the skin flaps to be created. Following crile's first radical attempt to use the ' $y$ ' incision for neck dissection other kinds of incision types have been described (crile 1906, martin et al 1951, schobinger 1957, mcfee 1960, stell \& brown 1970). As the understanding of the vascular supply of the neck skin and clinical practices improved some incisions started to be preferred, however no particular incision is used uniformly. The choice of incision type used for neck dissection depends on a number of factors. There are some fundamental specifications which are to be considered before placing a neck incision for neck dissection. Conditions that are to be provided for ideal incision: incision should provide a good exposure for surgery. Thus by exposing all the important structures of the neck, the block dissection of lymph nodes in the laterocervical region can be done. Incision should help in reaching the primary focus easier. It should not destroy the vascular supply of the skin flaps. It should protect the skin and also should prevent skin necrosis by supplying maximum blood flow to the flaps. It should look good cosmetically. It should not limit the movement of the head and neck by forming contractures related to the scar. It should not lead to complications during the post operative recovery period or these should be minimised. It should prevent the damage that can occur after the operation to the anatomical structures especially the carotid artery.

It should be capable of flap transfer for such cases as primary reconstruction or defect covering. It should be capable of tracheostoma and even pharyngostoma formation. It is necessary to know the vascularization of the neck skin to avoid complications such as carotid artery exposure, pharyngocutaneous fistulae and flap necrosis following neck dissection. Ellis 1963 demonstrated that the recovery of the neck skin is in a medial direction from the common carotid artery. Kambic and sirca 1967 stated that the arterial supply is in a vertical direction. According to this recovery of the skin on the anterior trapezius muscle is by descending branches of the facial and occipital artery while transverse cervical and supraclavicular arterial branches form the ascending branches. Rabson et al 1985 in their study on cadavers, established that the arterial supply of the skin of the neck is multifaceted and that there are four arterial branches which pass from the platysma muscle through to the top skin's surface. These platysma cutaneous arteries while supplying a particular region of the skin are also in anastomosis with each other. Ariyan 1986 states that these anastomosis remain intact during neck dissection while the platysma is dissected from the skin. Hetter 1972, freeland and rogers 1975 reported that there is alternative development of arterial supply even if facial, occipital and transverse cervical are ligated. The vasculature of the can be summarised into the upper neck region anterior to the angle of mandible are supplied by branches of facial and submental arteries. Occipital and external auricular branches of external carotid supply the upper lateral neck, the area between ramus of mandible and the sternocleidomastoid muscle. The transverse cervical artery and suprascapular artery provide vasculature to the lower half of neck. The transverse cervical artery should be preserved unless there is oncological indication for ablation. In addition there are large platysma-cutaneous branches and branches of superior thyroid supplying the front middle portion of the neck. ${ }^{6}$

\section{Classification Of Incisions}

The incision used in the dissection of the neck are generally classified into - vertical and horizontal. The combined procedure is also performed but the use of incisions in various directions will prevent sufficient arterial recovery of the flap. This increases the risk of flap necrosis in the tips of the flap. Transverse incisions 
have cosmetic advantages as they follow the natural skin folds of the skin. Recovery of the scar in these folds are rapid and successful. Attie 1957 supported this view and became the first to practice this type of incision. Transverse incision are also easy to modify. This type of incision destroys the neck's venous drainage from top to bottom and is major cause for flap separation (freeland and rogers 1975). Vertical skin incisions in comparison to transverse skin incisions are regarded to be disadvantages, because they intersect the natural skin folds of the skin and the vascular supply of the neck. ( attie 1957; mcfee 1960; stella and brown 1970; futrell and cheretien 1976 ). The vertical incisions tend to contract along their long axes. This leads to deformity and restricted action. This can be prevented by giving a posterior sigmoid curve to the vertical incision ( mcfee 1960; futrell and cheretien 1976).choosing a particular incision is important especially in the post irradiated neck area. In necks that have received radiotherapy vascular recovery in narrow angle flap's corners and sides may be reduced. The flap necrosis ensures and may expose the carotid artery. For this reason trifurcation and vertical incision should not be practised in necks which has received radiotherapy. Here transverse incisions are reported to yield good results (grillo and edmunds 1965; stella 1969; stella and brown 1970). The incisions used for neck dissection are: tri-radiate incision and its modification, hayes martin double ' $y$ ' incision, mcfee incision, apron flap incision, 'j' incision, hockey stick incisions \& its modification. ${ }^{7}$

\section{Tri-radiate incision \& its modification:}

Crile (1906) stated this type of incision which gave sufficiently wide exposure to the operating field. The disadvantages of the tri-radiate incisions are flap necrosis due to disruption of the blood supply. This incision has got a submandibular transverse incision and a longitudinal incision through the neck. The submandibular incision starts little beyond the midline near the lower border of the mandibular symphysis and it ends well back on the mastoid area, the two lines meet at the submandibular region at $120^{0}$. The vertical incision starts from where the two lines meet. In cases where curved horizontal incisions are used the incision continues as a smooth curve between the two extremities and the vertical incision starts from the lowest point of the curve. According to freeland \& rogers (1976) there is a horizontal watershed midway between the mandible and the clavicle. It will be ideal to place the horizontal incision below the carotid bifurcation to avoid carotid exposure when carotid blow out occurs. The vertical incision is placed little behind the carotid and continues down over the clavicle $3-4 \mathrm{~cm}$. This aids in wide exposure in the posterior triangle at the posterior-inferior angle. The skin flaps are raised subplatysmally. Advantage is that this incision provides good exposure to surgical site. The disadvantages are flap necrosis is high due to disruption of vasculature of skin flaps, occurrence of flap separation at the trifurcation site. ${ }^{6}$

\section{Modification of tri-radiate incision:}

Many modifications have been described. Schobinger (1957), cramer \& culf (1969) and conley (1970), schobinger suggested that the vertical limb instead of being straight should be curved posteriorly in order to avoid lying directly over the carotids. Cramer and culf suggested a "s" shaped vertical incision for the same. Conley suggested a posteriorly curving vertical incision rather than a horizontal incision. Here the incision starts from the submental region and ending by running downwards along the anterior border of trapezius to the level of clavicle gently curving posteriorly. The posterior part of the submandibular incision meets it at right angle approximately below the lobule. ${ }^{6}$

\section{Hayes martin incision:}

It is a paired "y" incision. Here the submandibular component is met by a vertical limb which below becomes continuos with an inverted "y" in the suprascapular region. Four flaps are thus created, the base of each extending to the limit of the neck dissection on each side. The posterior flap with no platysma at its base is liable to have less adequate blood supply than the other flaps. This flap most often gets cyanosed. The vascularity of the flaps can be put under less strain if the posterior flap is shortened by placing the vertical limb of the incision a little further back. Flaps can be broadened by raising the submandibular incision a little. The advantages and disadvantages are same as crile's incision but flap necrosis and carotid exposure is more in this type of incision.

\section{Mcfee incision:}

This incision differs from virtually all other incision as it avoids a vertical limb. Here two horizontal incisions are used one in submandibular region and other in the suprascapular region. Between these two a bipedicled flap is raised based anteriorly on the midline and posteriorly on the anterior border of trapezius. The flap is raised upwards to expose the lower part of neck until the dissection has proceeded far enough upwards to allow the resected specimen to be pulled through into the submandibular region. The advantages are excellent cosmetic result ( mcfee 1960, mcneil 1978), there is no lessening of vascularity in the centre of the flap (ariyan 1986), there is no angle intersection in incision (mcfee 1960 ), post operative wound recovery is rapid (mcfee), it is suitable in necks receiving radiotherapy and in peripheral vascular disease (maran et al 1989), recovery of the 
flap will not fail despite the cessation of the ascending and descending vascular recovery due to wide bipedicled flaps. ( stella \& brown 1970, daniel \& mcfee 1987 ). The disadvantages are the exposure is not good. ( hetter 1972), it is not suitable for bilateral simultaneous neck dissection ( chandler and ponzoli 1969), the operating period is long (mcfee 1960), posterior triangle dissection is difficult ( maran et al 1989, white et al 1993), difficulty may arise while working under the bridge flap, in short neck it might be difficult to distinguish between the front tip of the incision from that of the tracheostomy. ${ }^{6}$

\section{J incision:}

Eckert and byars 1952 practised the first forms of the " $\mathrm{j}$ incision. They extended the classical thyroid necklace incision laterally to the trapezius muscle and then slightly to the mastoid area. Grandon and brintnall 1960 popularised this type of incision. Acar et al 1999 reported excellent result in 320 cases. "j" incision has a vertical incision extending from the mastoid apex to $2 \mathrm{~cm}$ above the clavicle along the back of the sternocleidomastoid muscle, then it is extended in horizontal plane and continued parallel to the bottom edge of the cricoid cartilage and it is stopped at the point where the sternocleidomastoid muscle is attached to the clavicle. In situation that required bilateral neck dissection, the incision continued symmetrically and was extended across to the mastoid apex. The skin flap is elevated at the subplatysmal plane. The advantages are good exposure to surgical site, carotid artery is well protected, suitable in bilateral neck dissections. It can be modified easily less chance for occurrence of pharyngocutaneous fistula due to coverage of the top sutures used for closing hypophyranx and oesophagus. Flap vascularisation is good due to its posterior base. Suitable in necks which receive radiotherapy. Cosmetic result are good. Tracheostomy can also be performed with this type of incision in a horizontal segment. The disadvantages are contracture in the vertical top section. Flap separation in the horizontal flap section. ${ }^{6}$

\section{Hockey stick incision:}

Lahey et al 1940 first described a kind of hockey stick incision (his) for resection of thyroid gland carcinoma. It has been modified for radical neck dissection and functional neck dissection by eckert \& byars 1952; attie j. N. \& brooklyn n. Y. 1957; bocca e, pinataro o \& sasaki c t 1980 and ariyan 1986. Rush 1965 and gratz et al 1004 extended this incision to combine it with block resection of the oral cancer lesions. Hockey stick incision has got a longitudinal and transverse incision. The longitudinal portion runs from the mastoid process downward, 1-2 cm behind the anterior border of trapezius muscle and curves gently at the acromioclavicular junction. The transverse incision runs medially towards the sternum approximately $1 \mathrm{~cm}$ below the clavicular margin. This incision permits the elevation of a medio superiorly based skin flap. Bilaterally placed hockey stick incision permits the elevation of a superiorly based large skin flap and allows the deglovement of the whole neck. This is used when tracheostomy is planned along with bilateral neck dissection. In order to make incision for tracheostomy the transverse part of the incision is displaced superiorly and there by run across the anterior lower neck. This incision results in deglovement of the whole neck for providing access to the oral cavity. S. Omura et al 1999 reported good access and cosmetic result with reverse hockey stick incision for neck dissection and access to oral cavity while hockey stick incision provided good exposure and allowed tracheostomy to be performed through the same incision. The advantages are it has good exposure, it has good cosmetic result as the incision gets covered in the hair line and clothing, viability of the hockey stick incision flap is good. The disadvantages are access to submental area is not adequate. The flap has to be stretched and retracted in a upwardly direction and access to oral cavity is also not adequate. 7

\section{Reversed hockey stick incision:}

A reversed hockey stick incision was first described by schobinger as a long anterior skin flap for radical neck dissection and was extended to block resection of oral cavity by babcock \& conley and disanayaka. There is no three point trifurcation in hockey stick incision and reverse hockey stick incision and also they provide good exposure. Here the flap is raised inferiorly.here the incision is placed in submandibular region. The transverse incision curves gently $2-3 \mathrm{~cm}$ below the tip of mastoid process and runs medially towards the submental region approximately $2 \mathrm{~cm}$ below the lower margin of the mandible. Modifications of reverse hockey stick incision: upwardly extended reverse hockey stick incision - here the transverse incision of the reverse hockey stick incision is extended upward to the median of the lower lip resulting in the splitting of the lower lip. This provides excellent exposure to the operating field for the management of oropharyngeal cancer. Contra laterally extended reverse hockey stick incision - here the transverse incision is extended towards the conta lateral mastoid process. This incision is preferred for bilateral neck dissection (ipsilateral radical and conta lateral supraomohyoid neck dissection) and access to oral cavity. The advantages areiit provides good surgical exposure and gives better cosmetic result. The disadvantages are the descending arterial recovery may breakdown (hetter 1972) and the front and the tip of the medial flap might become necrotic however this weakest point is behind the carotid artery bifurcation. 
Apron flaps:

This flap was described by latyschevsky and freund 1960. Here only a horizontal incision from mastoid to mentum gently curving inferiorly up to upper border of the thyroid cartilage is used. While in bilateral neck dissection freund 1967 described an apron flap extending from one mastoid to other. According to freeland and rogers1975 study on the vasculature of the head and neck showed better safeguard of the vessels of neck flap in the superiorly based apron flap. The advantages are carotid artery is well protected, protects the descending arterial recovery. The disadvantages it will damage the ascending arterial and venous recovery, venous congestion and oedema might develop at the bottom corner. ${ }^{6}$

\section{Radical Neck Dissection}

This operation is defined as the en bloc removal of the lymph node bearing tissues on one side of the neck from the inferior border of the mandible to the clavicle and from the lateral border of the strap muscles to the anterior border of the trapezius muscle, included in the resected specimen are the spinal accessory nerve, internal jugular vein and the sternocleidomastoid muscle. The first description of this systematic en bloc removal of the lymphatics of the neck was published by crile in 1906. He stated that the removal of the internal jugular was essential due to intimate relationship of lymph nodes with it. The routine removal of the spinal accessory was advocated by blair and brown (1933), who believed that the nerve has to be removed to decrease the operating time and increase the certainty of total neck node removal. Martin (1951) championed the concept that a cervical lymphadenectomy for cancer was not adequate unless all the lymph node bearing tissues of one side of the neck were removed and that this was not possible unless the spinal accessory nerve, the internal jugular vein and the sternocleidomastoid muscle were included in the resection. Indications are in multiple clinically obvious cervical lymph node metastases, particularly when they involve lymph nodes of the posterior triangle of the neck and these are found to be closely related to the spinal accessory nerve, in large metastatic tumour mass or there are multiple matted nodes in the upper portion of the neck. The surgical procedure the initial step is to raise the skin flaps to the margins of the dissection that is the lower border of the mandible, the clavicle below the midline in front and the anterior border of trapezius behind, the anterior margin and the clavicular margin are readily defined. The submandibular skin flap requires to be raised with care to avoid damage to the marginal mandibular branch of facial nerve.

It runs in loose areolar layer deep to platysma and should be looked for, identified and traced out forward and backward out of the operating field. It is usual at this stage to divide the facial vessels as they cross lower border of the mandible. The submandibular prevascular and retrovascular lymph nodes are in close proximity to the nerve and they must be carefully dissected out from it. The posterior margin is much more difficult to define because of the consistency of the tissue filling the posterior triangle and the laxity of the trapezius muscle in anaesthetised patient. It is ideal to visualise the anterior border of the trapezius from end to end for subsequent clearance. Clearance of the neck is usually begun from the posterior triangle in the region of the posteroinferior angle. Here the floor is deeper than rest of the triangle and hence makes it difficult to dissect. Once beyond $3 \mathrm{~cm}$ above the angle on the posterior margin of the dissection, it is safe to deepen the plane to bare the prevertebral muscles. At this stage the posterior triangle of neck can be cleared up to the meeting point of sternocleidomastoid and trapezius at the upper angle of the triangle. In the process of this dissection about $5 \mathrm{~cm}$ above the clavicle the accessory nerve will be cut en passant. As the floor of the posterior triangle is cleared towards the posteroinferior angle the transverse cervical vessels are met passing across into trapezius that can be resected as a part of the dissection. Dissecting, along the lower border towards the posteroinferior angle, the tissue immediately above the clavicle is incised as far laterally at the trapezius exposing the sternocleidomastoid medially and passing more deeply laterally. Lateral to the sternocleidomastoid muscle, external jugular vein is ligated. Dissection further exposes the inferior belly of omohyoid that once divided, the dissection is continued deeply as far as the prevertebral fascia overlying the brachial plexus and the scalene muscles. Once the transverse cervical vessels are divided, the fat filling the posterior triangle is dissected towards off the prevertebral fascia. Now the floor of the triangle can be identified from occiput to the clavicle. The dissection can move forward on a broad front clearing each prevertebral muscles in turn. In order to keep the dissection moving uniformly forward along the entire length of the beck, it become necessary to section the sternocleidomastoid near its mastoid insertion. Dissection is continued to reach the anterior branch of cervical plexus as they emerge at segmental intervals just posterolateral to carotid sheath. Clearance of lower part of posterior triangle proceeds medially past the brachial plexus seen emerging between the two scalenes but safe behind the fascia until the dissections clearly impeded by sternocleidomastoid and this is then divided close to its sternal and clavicular insertions taking care to avoid damage to the internal jugular vein. Internal jugular vein is in close proximity to the sternocleidomastoid muscle at this point.

Once the sternocleidomastoid is divided dissection is proceeded medially over the scalenus anterior. Here it is important to watch carefully for the phrenic nerve which runs vertically across downward along the 
lateral line of muscle fibers. Once the nerve has been identified, dissection is carried on superficial to it, leaving it on the muscle, at the same time extending upwards so that its origin from the cervical plexus mainly $\mathrm{c}_{4}$ can be demonstrated. With its source visualized the branches of the plexus c 2 , c3 \& c 4 passing forward into the resection mass can safely be divided. Section of these branches coupled with forward traction brings the carotid sheath into view. Plane of dissection is along the prevertebral muscle behind carotid sheath. Just lateral to the carotid sheath over the scalenus anterior muscle thyrocervical trunk is seen giving thyrocervical artery which is ligated and dissected with the specimen. As the carotid sheath is approached the internal jugular vein with its bluish colour comes into view. The dissection plane here should stay close to the carotids. The vagus lying between artery and vein is dissected free and retained. The remaining tissue of the complex i.e. The internal jugular vein and deep jugular group is dissected forward. The absence of posterior branches of the internal jugular vein allows dissection to be carried up and down over much of its length without difficulty. Then the internal jugular vein is divided and double ligation using non-absorbable suture with one ligature transfixing the vein is a wise precaution. Now the dissection proceeds forwards and upwards baring the infrahyoid muscles, the anterior jugular vein divided and the ansa cervicalis sacrificed. As the dissection proceeds, superior and middle thyroid veins are divided. The superior belly of omohyoid is peeled off the infrahyoid group as part of the specimen and when dissection has reached the hyoid bone the muscle is divided at its origin. In the upper neck as the internal jugular vein is stripped off from the internal carotid artery the hypoglossal nerve comes into view (between the two). It is traced to the superior root of ansa cervicalis that is divided. Assuming ligation of internal jugular vein to precede, clearance of the submandibular triangle the dissection of vein free from hypoglossal and vagus nerve and the internal carotid artery is continued with forward and upward traction of the specimen until the transverse process of the atlas is reached. It is at this level that the internal jugular vein is divided. With the internal jugular vein mobilized any remaining fibers of sternocleidomastoid still attached to the mastoid process are divided until the digastric becomes visible, and the same level the lower pole of the parotid is sectioned in a line across to the angle of the mandible.

Here the retromandibular vein is ligated. Downward retraction of the sternocleidomastoid and the parotid pole brings digastric into view and depending on the local condition it can either be retracted or divided watching for the occipital artery in so doing. Beneath the digastric the internal jugular vein is exposed, doubly ligated and divided in the same way as the lower end. Clearance of submandibular triangle is now began. The upper border of the triangle is defined by deepening the dissection along the lower border of the mandible to the bone in front of the masseter. Here the facial vessels are ligated and divided as they cross the lower border just in front of the masseter if this has not already been done. At the angle of the mandible this line of section becomes continuous with the section line of the parotid. The inner surface of the mandible below the mylohyoid line has no attached structure in front of the medial pterygoid muscle and a finger inserted medial to the mandible can mobilize the soft tissues off the bone. Stripping is pursued upward to the insertion of the mylohyoid. In the symphyseal area clearance of the digastric muscle should be extended across the midline as far as the contralateral digastric muscle, making it possible to clear the submental nodes. Tissue between the anterior bellies of digastric is dissected off the midline raphe of mylohyoid. If posterior belly of digastric has been sectioned the entire digastric is removed as a part of the resection specimen and the dissection plane continues on the mylohyoid. If digastric is retained dissection proceeds around it returning behind it to mylohyoid. In this part of dissection mylohyoid is a key structure. The tissue resected off mylohyoid is recognized as the superficial lobe of the submandibular salivary gland with its duct. Above and parallel to its duct lies and lingual nerve. Below and parallel to the duct runs the hypoglossal nerve with hypoglossal venous plexus. The duct is freed from its surroundings, ligated and divided and the gland is then dissected completely off hypoglossus. In so doing facial artery, looping over the gland is exposed for ligation and division. Final dissection cleans the specimen from any minor remaining attachments. De santo et al 1985; leemans et al 1990 reported a 3 to $7 \%$ recurrence rate in the neck following a radical neck dissection with histologically tumour free nodes. Strong 1969 reported an ipsilateral recurrence rate of 20 to $71 \%$ following a therapeutic radical neck dissection for clinically and histologically nodes. This series included large number of cases of uncontrolled primaries. He also found a recurrence rate of $36.5 \%$ in patients with histologically single node and $71 \%$ in histologically multiple positive nodes. Johnson et al 1981, snow et al 1986 and carter et al 1985 have shown that the recurrence rate in the neck after radical neck dissection is significantly higher when extracapsular spread of tumour is demonstrated. Carter et al 1985 also found that macroscopic extracapsular extension is associated with a higher risk for recurrence (44\%) whereas microscopic extracapsular spread has a lesser recurrence rate $(25 \%)$ similar to intracapsular node metastases $(32 \%)$.

Snow et al 1986 has shown that patients with four or more histologically positive nodes and extracapsular spread have a significantly greater likelihood for distant metastases $(>60 \%)$ than patients with one histologically positive node with extracapsular spread $(<30 \%)$. Efficacy of radical neck dissection is to be considered with adjuvant radiotherapy. Leemans et al 1990, strong 1969, vikram et al 1984 reported a reduction in neck metastases by the addition of radiation therapy to radical neck dissection. However de santo et al 1985 
in a review of 1192 neck dissections reported no difference in the probability of recurrence in the neck patients with $\mathrm{n} 2$ neck disease treated by dissection alone, preoperative radiation, postoperative radiation or miscellaneous radiation. ${ }^{1,3}$

\section{Modified Radical Neck Dissection}

Modified radical neck dissection with preservation of spinal accessory nerve (type i). A modified radical neck dissection with spinal accessory nerve preservation is defined as the enbloc removal of the lymph node bearing tissues of one side of the neck from the inferior border of the mandible to the clavicle and from the lateral border of the strap muscles to the anterior border of trapezius preserving the spinal accessory nerve. The internal jugular vein and sternocleidomastoid muscle are resected with the specimen. Rationale: the following observation resulted in exploring alternative cervical neck dissection. The morbidity associated with the radical neck dissection, especially the shoulder disability that results from the resection of the spinal accessory nerve, and to a lesser extent the cosmetic deformity that results from this operation especially when done bilaterally. The realisation that the spinal accessory is not in close proximity to the nodes involved by tumour and that its preservation does not compromise the oncological soundness of the operation. Indication is when there is clearly identifiable plane of dissection between the tumour and the nerve. The incisions are the same as that of radical neck dissection. After the flaps are raised, the spinal accessory nerve is exposed as it crosses the anterior border of the trapezius at about $2 \mathrm{~cm}$ above the clavicle, then the nerve is exposed through the posterior triangle incising the fascia and fatty tissue over a hemostat. This is continued through the upper portion of the sternocleidomastoid muscle, exposing the nerve in its entire course through the neck. The nerve is freed from underlying tissue. Rest of dissection is carried out as routine radical neck dissection. Alternatively, the nerve can be identified high in the neck, medially to the posterior belly of digastric where it lies either posterior or lateral to internal jugular vein. From here it is exposed and isolated. Isolation of the nerve is easy if bleeding is kept to a minimum. Dargent \& papillon 1945 and skolnik et al 1967 were among the first to advocate the spinal accessory nerve preservation.

Subsequently this was used by roy \& beahrs 1969 , carenfelt \& eliasson 1980, brandenburg \& lee 1981, chu \& strawitz 1978, pearlman et al 1982. The recurrence rate following modified radical neck dissection was 4 $\%$ to $7 \%$ similar to radical neck dissection of $3 \%$ to $7 \%$. Functional, conservative or conservation neck dissection (type iii). This operation is defined as the en bloc removal of the lymph node bearing tissue of one side of the neck including the nodes in level $\mathrm{i}$ to $\mathrm{v}$, preserving the spinal accessory, the internal jugular vein and the sternocleidomastoid muscle. The submandibular gland may not be removed. Rationale: suarey 1963 discussed the rationale for this type of neck dissection. He stated that it is oncologically safe to remove lymph nodes of the neck without sacrificing the sternocleidomastoid muscle, the internal jugular vein and the spinal accessory nerve. Since in necropsy of patients with head and neck cancer he noted that lymph nodes were always found in the fibrofatty tissue either away or near blood vessels, particularly veins, lymph nodes are not part of adventia of vessels. He also observed that lymph nodes are not located within the muscular aponeurosis of the salivary gland capsule. I.e. In sternocleidomastoid muscle and submandibular gland. But lymph nodes are found within parotid. There is no surrounding communication of lymphatics with the surrounding fascia or muscle. Saurez demonstrated its feasibility in 275 cases. This operation was popularised by bocca who coined the term functional, conservative or conservation neck dissection. Bocca 1980 emphasised the presence of muscular and vascular aponeuroses of the neck, which define compartments filled with fibrofatty tissue. The lymphatic system of the neck, contained within the compartments can be excised in an anatomic block by stripping the fascia off the muscles and vessels. Indications are in cases of primary in larynx and hypopharynx where the submandibular triangle is at low risk of containing metastases.in differential carcinoma of the thyroid. Contraindication is in cases of node fixation. The procedure involves dissection in submandibular region. Then the fascia over sternocledomastoid is dissected beginning from posterior to anterior border. Muscle is retracted posteriorly and spinal accessory nerve is identified at the upper $1 / 3^{\text {rd }}$ and lower $2 / 3^{\text {rd }}$ of muscle. Fibrofatty tissue with lymphatic tissue are dissected away from spinal accessory nerve. The dissection is carried over the splenius capitis and levator scapulae muscles in the posterior triangle. The superficial layer of deep cervical fascia is divided at the junction of sternocledomastoid and anterior border of trapezius. Here the external jugular vein is clamped and divided. Also the omohyoid muscle is divided. Then the specimen is brought forward from posterior to sternocledomastoid muscle. The specimen is then dissected of carotid sheath and internal jugular vein while taking care not to injure the thoracic duct. ${ }^{1,3}$

\section{Selective Neck Dissection}

It involves selective en bloc removal of only lymph node groups of neck depending on the location of the primary tumour which are most likely to contain metastasis. The supraomohyoid neck dissection consist of the removal of nodal regions i. Ii and iii. In expanded supraomohyoid neck dissection region iv are also removed. The lateral neck dissection consist of en bloc removal of nodal regions ii, iii and iv. The 
'posterolateral neck dissection' consist of removal of suboccipital and retroauricular lymphnodes and nodes from regions ii, iii, iv and v. Rationale: medina \& byers 1989 stated that en bloc removal of the nodes at highest risk for metastasis is anatomically justified and it has the same therapeutic value as radical or modified neck dissection. It is associated with less post operative morbidity. Rouviere 1938 and fish \& siegel 1964 showed that lymphatic drainage of the mucosal surface of head and neck flow in a predictable routes. Lindberg 1972 demonstrated the jugulodigastric and midjugular nodes are most frequently involved nodes in patients with carcinoma of the oral cavity. Further he noted submandibular nodes are involved in carcinoma floor of mouth, anterior oral cavity, tongue and buccal mucosa. Skolnik 1976 in a study of radical neck dissection specimens noted no radical involvement of posterior cervical triangle. Shah 1990 in a retrospective study of 119 radical neck dissection found that oral cavity tumours metastasised to regions i, ii and iii nodes, whereas carcinoma of oro, oropharynx and larynx involved mainly region ii, iii and iv nodes. The supraomohyoid neck dissection (sohnd) is the most commonly performed selective neck dissection for the treatment of the n0 neck. It involves the en bloc removal of cervical lymph node groups i-iii.

The posterior limit of this dissection is marked by the cutaneous branches of the cervical plexus and the posterior border of the scm. The inferior limit is the superior belly of the omohyoid muscle where it crosses the ijv. Indications are SOHND is indicated in patients with primary tumors arising from the oral cavity without clinical or radiologic evidence of cervical metastasis but who have a high probability of occult lymphatic disease. The oral cavity includes the area between the vermillion border of the lips and the junction of the of the hard and soft palate superiorly and the circumvallate papillae of the tongue inferiorly. Subsites in the oral cavity include the lips, buccal mucosa, upper and lower alveolar ridges, retromolar trigone, hard palate, and anterior two thirds of the tongue, and floor of mouth. Medina recommends sohnd in patients with staged $\mathrm{t} 2 \mathrm{-t} 4 \mathrm{n} 0 \mathrm{or}$ txn1 when the palpable node is less than $3 \mathrm{~cm}$, clearly mobile, and located in levels i or ii. Bilateral sohnd is indicated in patients who have carcinomas of the anterior tongue or oral tongue and floor of mouth that approach or cross the midline. Sohnd is indicated along with parotidectomy in patients with squamous cell carcinoma, merkel cell carcinoma, or selected stage i melanomas (thickness between 1.5 and $3.99 \mathrm{~mm}$ ) in the cheek and zygomatic regions of the face. The lateral type neck dissection refers to the en bloc removal of the jugular lymph nodes including levels ii-iv. Indicated in patients with tumours of larynx, oropharynx and hypopharynx staged t 2 to $t 4$ $\mathrm{n} 0$ or $\mathrm{n} 1$ or $\mathrm{t} 1 \mathrm{n} 1$ when palpable node is located in level $\mathrm{i}$ or ii. This procedure is usually done bilaterally as the lymphatics drainage metastases bilaterally. The posterolateral type neck dissection involves the en bloc excision of lymph bearing tissues in levels ii-iv and additional node groups including the suboccipital and postauricular node. This type of neck dissection is primarily used to treat the neck in patients with cutaneous malignancies (primarily squamous cell carcinoma, melanoma, and other skin tumors with metastatic potential, such as the merkel cell carcinomas) and soft tissue sarcomas. The anterior compartment neck dissection involves the en bloc removal of lymph structures in level vi.

The lymph node groups excised are the perithyroidal nodes, pretracheal, precricoid (delphian), and paratracheal nodes located along each recurrent laryngeal nerve. The superior limit of the dissection is the hyoid bone and the inferior extent is the suprasternal notch. Laterally the dissection is carried out to the carotid sheath. Indicated in (1) selected cases of differentiated thyroid carcinoma, (2) parathyroid carcinoma (3) subglottic carcinoma (4) glottic carcinomas with subglottic extension, (5) cervical esophagus. Robbins (1998) also recommends this neck dissection in some patients with hypopharyngeal carcinoma as paratracheal nodes are at high risk for occult disease in these tumors. A unilateral neck dissection is usually performed through a apron like incision that extends from the mastoid tip to mandibular symphysis. The lowest point of the incision is usually located at the level of the thyrohyoid membrane. The incision can be modified to include a lip splitting incision or a descending limb can be added for added exposure. To perform a bilateral dissection an apron like incision is made extending from one mastoid to other overlying the thyrohyoid membrane. A superior flap is raised in a subplatysmal plane up to the inferior border of the mandible. The inferior flap is raised to about 1 inch above the clavicle, however the inferior flap can be raised up to the clavicle. The dissection begins in the submental region. The lymphatic fibrofatty specimen is pulled inferiorly and laterally away from the digastric muscle and the mylohyoid. The submandibular gland with lymphnodes is dissected next away from the mandible. The fascia overlying the posterior belly of digastric and omohyoid is incised. The dissection is carried out in the area posterior to omohyoid preserving the hypoglossal and superior thyroid artery. Then the posterior dissection is began from the anterior border of sternocledomastoid muscle. The fascia is retracted anteriorly and dissection is carried around the muscle up to the point where the spinal accessory nerve enters the sternocledomastoid muscle. The nerve is carefully dissected free from the surrounding structures. Above the level of the nerve, splenius capitis and levator scapulae the dissection is completed. The dissected specimen in this region is brought forward. The dissection is carried along the carotid sheath. The inferior level of dissection is usually to the level where the omohyoid crosses the internal jugular vein. If nodes are found in level iii then the dissection is extended to level iv below the omohyoid sacrificing the omohyoid muscle. This operation is called "extended supraomohyoid neck dissection". The neck dissection is done similarly on the opposite side. 
When this operation is complete only small amounts of lymph nodes are left behind in the posteroinferior aspect of the neck. ${ }^{1}$

\section{Extended Neck Dissection}

Any of the neck dissections described above can be extended to remove either lymphnode groups or vascular neural or muscular structures not routinely removed in a neck dissection. Neck dissection can be extended to remove retropharyngeal lymphnodes in primaries of pharyngeal wall. These lymphnodes are involved in tumours of base of tongue, tonsil, soft palate and retromolar trigone. Paratracheal and pretracheal nodes are removed in carcinoma of transglottis and subglottis, cervical oesophagus, trachea and thyroid carcinoma. Adequate removal of a metastatic tumour in the neck may dictate the need to extend a neck dissection to resect structures such as hypoglossal nerve, levator scapulae muscle or carotid artery. Controversies exist over the ligation of internal and common carotid artery. Byers and ballantyne 1985 noted that prognosis of a patient with neck disease which warrants resection of carotid arteries is dismal and it is not justified to remove carotid arteries. Moore and baker 1955 observed a 30\% mortality rate and $45 \%$ cerberal complication rate among patients who underwent carotid artery ligation. Goffinet et al 1985 reported encouraging results with large cervical metastasis attached to carotid artery who were treated by resection of tumour and intraoperative iodine 125 seed was placed using vicryl tubes. He showed a $77 \%$ tumour control but $1-$ yr. Survival rate was $15 \% .^{3}$

\section{Bilateral Radical Neck Dissection}

This procedure is not often performed. The loss of both sternocleidomastoids makes it difficult for all patients, impossible for some to lift the head from a recumbent position without using the hands to support it. The loss of both accessory nerves doubles the usual disability from this source. Bilateral dissection is most often carried out as two separate operative procedures separated in time when metastatic nodes develop on the contralateral side of the neck after a node dissection on the ipsilateral side. The modifications in bilateral dissections concern the skin incisions and the management of internal jugular vein. If there is large interval between the neck dissection the skin incisions can be the one used for a single neck dissection. When the procedures are simultaneous or the gap in time is very short, the problem changes, because the anterior flap raised in most dissection incisions ceases to have a base if it is raised on both sides at once. Duplicated conley variant of triradiate incision can be used in these circumstances. This has the most assured blood supply. This can be modified from a curve into a series of straight line and in this form has been recommended (lore, 1973). The internal jugular vein is the main venous connection between the brain and the heart and its simultaneous loss on both sides has dramatic and undesirable effects. These effects are mitigated if it is possible to ablate the veins at intervals rather than simultaneously.

Compensatory veins appear to open up which are efficient enough to prevent the more undesirable changes and the process of compensation seems to occur with surprising speed. An interval of four weeks is usually adequate though naturally the safe time gap is likely to vary some what in different patients and one would make it as long as possible, as well as giving consideration to retaining one vein if the time interval was short. With any second neck dissection preservation of the vein is worth attempting and for shorter time lag from first neck dissection. In the extreme situation of a bilateral simultaneous neck dissection can be done with one vein retained if at all possible. An attempt at preservation of vein made on the first side dissected leaves the second variable for another attempt, should the first be unsuccessful. Morfit and perzik 1952 reported a case of bilateral simultaneous neck dissection without vein preservation. The patient developed massive edema and cyanotic swelling over face which subsided in due course of time. Assuming preservation of at least one internal jugular vein, this procedure would result in virtually no disability and used in the correct clinical situation is likely to be equally effective. 1,3

\section{Intraoperative Complications Of Neck Dissection}

In addition to various medical complications that can occur following any surgical procedure, there are a number of surgical complications associated with neck dissection. The complications can be divided into intraoperative and postoperative complications. Complication of cerberovascular accident is also common following head and neck dissection. It is important to manage this properly otherwise it might result in neurological deficit or death. Sobol et al 1982, have described five pathological mechanism for cerberovascular complications following head and neck surgeries. Embolism from ulcerated plaque, intravascular thrombosis with occlusion, unintentional surgical ligation, transection or laceration of the carotid artery, ligation of the external carotid artery with previous internal artery occlusions., transient reduction in cerberovascular profusion pressure. Intraoperative complications are these are mainly vascular complications which include bleeding (arterial / venous), chyle leak and nerve injury. Inadvertent arterial injury might result in serious bleeding. Most serious complications are vascular in nature. Most dramatic injury is to the common carotid artery and its 
branches which occurs rarely during an orderly neck dissection. If bleeding is from the external carotid artery or one of its branches the vessel it may be ligated. The carotid system contribute to $85 \%$ of cerebral blood flow, if occluded collateral flow must develop from the circle of willis. Moore \& baker 1955 found a difference in effects following elective and nonelective ligation of the common carotid artery. In 69 patients following elective ligation $40 \%$ developed cerebral complication and $17 \%$ died while in 87 cases of nonelective ligation $88 \%$ developed cerebral complication and $38 \%$ died. Ledgerwood et al (1980) compared primary repair versus ligation of carotids and reported excellent results with primary repair. Small lacerations are repaired. In moderately large loss of artery end to end anastamosis is planned. Lacerations of internal carotid artery close to the base of skull are best ligated. When tumour invades the internal carotids, the artery can be repaired with saphenous vein graft with synthetic suture materials. Fleming and petrie (1968), miller and bergstorm (1974) reported cases of internal carotid thrombus. In suspected cases of thrombus thrombectomy is performed and if not viable ligation of internal carotid artery is considered. Bleeding from internal jugular vein may be potentially more dangerous than arterial bleeding as it is difficult to control. A most dangerous injury is at the junction of internal jugular and subclavian veins.

To prevent air embolism patient may be brought down to level position from an elevated position. Hemorrhage from the proximal aspects of internal jugular vein is less dangerous. If proximal control cannot be obtained with clamps and ligature, then the jugular foramen may be packed with oxidized cellulose. Alternatively mastoid tip may be removed in an effort to gain direct control. Usually prevented by isolating the lymphatic pedicle between the carotid artery and the phrenic nerve and clamping this pedicle prior to dividing and ligating it. Intraoperative chylous leak should be recognized as opalescent fluid in posterior and inferior aspect of the dissected neck. Once it has been isolated it should be clamped and secured with a tie. The spinal accessory, cervical, cutaneous and great auricular nerves are intentionally sacrificed during standard radical neck dissection. If spinal accessory nerve is accidentally injured as in modified neck dissection, an interpositional nerve graft of greater auricular nerve can be used for repair. This can decrease the shoulder disability. Injury to motor branches of cervical plexus can also result in shoulder disability. Injury to phrenic nerve can occur without its transection. If the phrenic nerve is transected it should be reapproximated. Injury to the cervical contribution to the phrenic nerve may be avoided by dissecting the neck from the posterior triangle anteriorly and transecting the cervical cutaneous nerve, distal to their phrenic contribution. Injury to brachial plexus is uncommon and may be avoided by undermining the posterior triangle of the neck and visualizing the entire brachial plexus prior to transection or to clamping of the posterior triangle fat. Injury to vagus nerve will result in ipsilateral hypopharyngeal and vocal cord anesthesia or vocal cord paralysis. No cardiac or git dysfunction is known to result from transection of the vagus. Unilateral loss of hypoglossal nerve function results in little disabilities. Bilateral loss of function may occur in laryngectomy with resection of base of tongue cancer and would result in severe crippling of the swallowing function. Injury to cervical sympathetic chain results in horner's syndrome. Paralysis of facial nerve is an unusual complication. Care must be taken at the time of sacrifice of posterior belly of digastric since the stylomastoid foramen through which the facial nerve emerge is located at the posterior superior aspect of the posterior belly. The facial nerve may be identified and dissected free. Unilateral sacrifice of marginal mandibular branch of facial nerve may be necessary when there is obvious nodal metastases in the upper neck. This results in little disability and minimal cosmetic deformity. However loss of function of both marginal mandibular nerves does result in incompetent oral commissure. ${ }^{2}$

\section{Postoperative Complications}

Haematoma: it is one of the more common complications that predispose to wound infection, flap necroses, carotid exposure and fistula formation. To prevent haematomas meticulous hemostasis should be attained and prior to closure at least two medium drainage catheter should be placed in the supraclavicular fossa and posterior triangle. Two other catheters should be placed anterior to the carotid artery and adjacent to the pharyngeal or oral cavity closure in composite resections. Constant suction should be maintained during the early post-operative and bulky external compression drainage applied. Chylous fistula: Rarely a chyle leak will be noted as a bulge beneath the skin of the supraclavicular fossa. In addition to elevation of skin flaps, the chylous leak produces induration edema and erythema of the overlying skin. A chylous fistula may lead to chylothorax that mimics pleural effusion. This may require chest tube drainage. Bilateral neck dissection produces complications related to venous and lymphatic obstruction. Airway obstruction occurs frequently. Increased intracranial pressure may occur, that can lead on to cerebral edema, impaired neurologic function, and blindness stroke. ${ }^{3}$

\section{Sequelae Of Radical Neck Dissection}

Shoulder dysfunction: this is due to loss of trapezius function when spinal accessory nerve is cut. Participating muscles that compensate for loss of trapezius function are scapulae, rhomboidus and levator. Abduction of shoulder becomes limited. Patient try to compensate by contralateral flexion of the trunk. 
Enlargement of sternoclavicular joint: this is partly due to prominence of the head of the clavicle following removal of sternocleidomastoid. Stress fracture of the clavicle. Gustatory sweating: this is due to aberrant regeneration of autonomic nerve fibres following injury. The most typical area is submandibular area on the upper cervical flap. Sensory loss: this is due to sectioning of branches of cervical plexus. The area of sensory loss can involve the entire neck, chest from midline to just below the insertion of the deltoid laterally and inferiorly to the nipple line, posteriorly across the scapular spine, superiorly to the occiput including the entire ear. Patient should be advised not to expose this denervated skin to extremes of temperature. Neuromas: neuromas of the cutaneous roots of cervical nerves are common and may cause pain, tenderness, hyperesthesia. Graham in his review of 50 patients could find no correlation between the occurrence of neuroma and the use of preoperative or postoperative irradiation or a particular type of cervical flap. He found no recurrence of neuromas after treatment by resection high ligation and local instillation of triamcinole. Scar contracture: significant scar contracture of the cervical flap incisions may occur if wound dehiscence has occurred or if the vertical limb of a cervical incision is placed more than $4 \mathrm{~cm}$ anterior to the border of the trapezius muscle. Multiple z plasty will remove the tension of the scar contracture but will not improve the cosmetic appearance. 1,3 .

\section{Conclusion}

A precise knowledge of the surgical anatomy is essential for neck dissection. Neck dissection is an effective procedure for the management of diseases of neck. This is an ideal treatment for management of malignancies and metastasis to the head and neck region. For management of no neck refined surgical techniques like selective or functional neck dissection have been developed in order to reduce the morbidity associated with radical procedures.

\section{References}

[1]. Charles w. Cummings, john m. Fredrickson, lee a. Harker, charles j. Krause, david e. Schurller. Neck dissection. Otolaryngologyhead and neck surgery. Vol. Ii, $2^{\text {nd }}$ edition. 1993: 1649-1672.

[2]. Ian a. Mcgregor, frances m. Mcgregor. Neck dissection. Cancer of the face and mouth - pathology and management for surgeons. Churchill livingstone.1986: 282-320.

[3]. Ian t. Jackson. Inrtra oral tumour and cervical lymphadenectomy. Grabb \& smith's plastic surgery. Sherrel j. Aston, robert w. Beasley, charles h. M. Thorne. $5^{\text {th }}$ edition. Lippincott- raven . $1997: 439-452$

[4]. L. H. Sobin \& ch wittekind. Tnm classification of malignant tumours. $5^{\text {th }}$ edition. Uicc, a john wiley \& sons inc. Publication. 1997.

[5]. P. Hermanek, r. V. P. Hutter, 1. H. Sobin \& ch wittekind. Tnm atlas. Illustrated guide to the tnm / ptnm classification of malignant tumours. $4^{\text {th }}$ edition. Springer. 1997.

[6]. Aydin acar, gürsel dursun, ömer aydin,yücel akbaş. J incision in neck dissections. The journal of laryngology and otology. 1998: 112: $55-60$

[7]. Susumu omura, hiroki bukawa, ryoichi kawabe, shinjiro aoki, kiyohide fujita. Comparision between hockey stick and reverse hockey stick incision: gently curved single linear neck incisions for oral cancer. Int. J. Oral maxillofac. Surg.1999:28:197-202. 